\title{
Growth of stylolite teeth patterns depending on normal stress and finite compaction
}

*Koehn, Daniel; **Renard, François; ***Toussaint, Renaud; *Passchier, Cees W.

*Tectonophysics, Institute of Geo sciences, University of Mainz, Becherweg 21, 55099 Mainz, Germany, email: koehn@uni-mainz.de

**LGIT-CNRS-Observatoire, Univ ersité Joseph Fourier BP 53, F-38041 Grenoble, France \& Physics of Geological Processes, University of Oslo, Norway

***Institut de Physique du Globe de Strasbourg, UMR CNRS 7516, 5 rue Descartes, F-67084 Strasbourg Cedex, France

\section{Abstract}

Stylolites are spectacular rough dissolution surfaces that are found in many rock types.

They are formed during a slow irreversible deformation in sedimentary rocks and therefore participate to the dissipation of tectonic stresses in the Earth's upper crust. Despite many studies, their genesis is still debated, particularly the time scales of their formation and the relationship between this time and their morphology.

We developed a new discrete simulation technique to explore the dynamic growth of the stylolite roughness, starting from an initially flat dissolution surface. We demonstrate that the typical steep stylolite teeth geometry can accurately be modelled and reproduce natural patterns. The growth of the roughness takes place in two successive time regimes: i) an initial non-linear increase in roughness amplitude that follows a power-law in time up to ii) a critical time where the roughness amplitude saturates and stays constant. We also find two different spatial scaling regimes. At small spatial scales, surface energy is dominant and the growth of the roughness amplitude follows a power-law in time with an exponent of 0.5 and reaches an early saturation. Conversely, at large spatial scales, elastic energy is dominant and the growth follows a power-law in time with an exponent of 0.8 . In this elastic regime, the roughness does not saturate within the given simulation time.

Our findings show that a stylo lite's roughness amplitude only captures a very small part of the actual compaction that a rock experienced. Moreover the memory of the 
compaction history may be lost once the roughness growth saturates. We also show that the stylolite teeth geometry tracks the main compressive stress direction. If we rotate the external main compressive stress direction, the teeth are always tracking the new direction. Finally, we present a model that explains why teeth geometries form and grow non-linearly with time, why they are relatively stable and why their geometry is strongly deterministic while their location is random.

\section{Introduction}

Dynamic roughening of interfaces is an important research topic in many scientific discip lines. A natural and spectacular example of such processes are stylolites, pairs of dissolution surfaces facing each other, that are found in many rocks, mostly in limestones, and which are often used for ornamental stone. A characteristic feature of stylolite interfaces is their pronounced roughness with "teeth"- or "pen"-like geometries [stylus = pen, 1-5]. The dark residual material that is collected within the stylolite consists mostly of clay particles [6]. Stylolites have mainly been described qualitatively in Earth Sciences so that dynamic models of their growth and scaling properties are only now emerging [6-8]. However, the development mechanism of their teeth-geometry, with peculiar square peaks (Fig. 1), is up to now not understood.

Stylolites tend to grow perpendicular to the maximum compressive stress direction, and exhibit a pronounced roughness on several scales [3]. Teeth like peaks develop with sides or iented sub-parallel to the maximum stress direction (Fig. 1)[ 9]. The orientation of stylolites and their teeth is commonly used by geologists as an indicator for the direction of the maximum compressive stress [10-12] and the amplitude of the stylolite roughness is sometimes used as a direct estimate of the compaction that the rock underwent [13]. We use compaction here not only for a reduction in porosity of a rock but also for a vertical shortening of the rock due to the weight of the overlying sediments. Studies on the validity of these "rule of thumb" methods of compaction estimates are clearly missing, and the reason for 
this is probably the complexity of the development of stylolites. Since stylolites are so complex, there is also an ongoing debate in the literature about ways to classify them.

These features grow as a function of physical and chemical interactions and it is not easy to study them experimentally [14] nor in numerical models [6]. However, experiments or numerical models are needed since field observations alone cannot elucidate the dynamics of the roughening process but only represent snapshots at a given time. Early works in Earth Sciences classify stylolites according to their shape using qualitative methods [3]. However, this classification cannot successfully encompass all stylolite patterns, which can be found in numerous rock types and on a large range of scales [1-3]. Also, these classifications are often not related to any specific material parameters, scaling properties or growth conditions. More promising methods use qualitative descriptions of the stylolite roughness suggesting that they are fractal surfaces $[7,15]$ and self-affine structures $[6,8,14]$. Here we present new microdynamic simulations of stylolite roughening that allow us to explore the dynamics of the stress induced roughening process in time and space, illustrate stylolite geometries that develop at different scales and propose a process-based explanation for the growth of stylolite teeth. These simulations are based on first principles of physics and chemistry, without any ad hoc phenomenological equation. It should also be underlined that contrarily to previous linearized models $[6,8]$, the model used here allows the exploration of the fully developed structures, i.e. they take into account both, the non-linearities of the system that can be associated to large departures from flat surfaces and the solid-solid contacts occurring through the stylolite.

\section{The numerical model}

We start with an initially flat interface where dissolution can take place and the solid can only dissolve and not precipitate. This would be the case with an undersaturated fluid facing the solid. This flat interface may represent an initial "anticrack", that is assumed to represent the stylolite at an early stage of formation $[16,17]$. The model is two-dimensional 
and it assumes that everything that dissolves along the interface is transported instantaneously in the fluid out of the system, i.e. that diffusion in the fluid pocket is not limiting the process, but happens at a much faster time scale than the dissolution itself. Accordingly, the fluid has a constant solute concentration. The model is part of the modelling environment "Elle" [18]. The setup of the model is as follows: two solids are pressed together with a confined fluid layer in between them (Fig. 2). The right and left hand side of the model are fixed through elastic walls whereas the lower and upper walls of the model are moved inwards at a constant displacement rate. The solid is made up of small particles that are connected via linear elastic springs along a hexagonal lattice. These elements can represent either a single grain or a pack of smaller grains. At a scale larger than the grains, this network behaves in a classical elastostatic way.

\subsection{Thermodynamics and kinetics of stylolite dissolution}

Dissolution of the solid takes place in small steps dimensioned in such a way that one element of the solid is dissolved at every step. Dissolution follows a simple linear rate law

$$
D=k V\left[1-\exp \left(-\frac{\left\{\Delta \psi+\Delta \sigma_{n}\right\} V}{R T}\right)\right],
$$

where $D$ is the dissolution velocity of the interface $\left(\mathrm{m} \mathrm{s}^{-1}\right), k$ a dissolution kinetics rate constant $\left(\mathrm{mol} \mathrm{m}^{-2} \mathrm{~s}^{-1}\right), V$ the molecular volume of the solid $\left(\mathrm{m}^{3} \mathrm{~mol}^{-1}\right), R$ the universal gas constant $\left(\mathrm{J} \mathrm{mol}^{-1}{ }^{\circ} \mathrm{K}^{-1}\right), T$ the temperature $\left({ }^{\circ} \mathrm{K}\right), \Delta \psi\left(\mathrm{J} \mathrm{m}^{-3}\right)$ the changes in Helmholtz free energy of the solid during dissolution of a solid element, and $\Delta \sigma_{n}(\mathrm{~Pa})$ the normal stress gradients along the interface [further details of the derivation are given in 19,20]. The Helmholtz free energy takes into account the variations in elastic and in surface energy. Changes in surface energy are calculated from the curvature of the interface. The surface energy $\left(E^{s}\right)$ per area unit around a single element located at the interface can be expressed as 


$$
E^{s}=\frac{\gamma}{\rho}
$$

110 where $\gamma$ is the surface free energy and $\rho$ is the local radius of curvature of the interface. The

111 local curvature is determined using the two neighbours of each element along the interface.

112 The sign of the curvature $\rho$ is such that it is driving dissolution in the rate law when the solid

113 is convex towards the fluid, and precipitation when it is concave. We perform an average

114 across the interface using the local surface energies of elements and those of their neighbours

115 (up to $\mathrm{n}=40$ in each direction) with the expression

$$
E_{i}^{a v s}=\frac{1}{C} \sum_{h=1}^{n}\left(\frac{1}{(2 h+1)^{2}} \sum_{j=0}^{2 h+1} E_{i+j-h}^{s}\right)
$$

117 where $E_{i}^{a v s}$ is now the average surface energy for element $i, E_{i}^{s}$ the local surface energy for element i, and $E_{i+j-h}^{s}$ surface energies of neighbouring elements along the interface, and

$119 C=\frac{\pi^{2}}{4}-\frac{17}{9} \approx 0.578512$ is a normalization factor that ensures that the average surface energy on all sites is preserved in this averaging procedure. The local surface energies are divided by the sum of added elements and their maximum distance (divided by the initial radius of curvature of a particle). This av eraging procedure amounts to consider a coarsegrained surface energy, at the scale of a few elements, and allows to avoid artefacts that could arise from the discreteness of the model. In this way, a singular local term $E_{i}{ }^{s}$ obtained at a corner of the interface is smoothened for $E_{i}^{\text {avs }}$ over a neighbourhood of a few grains. This procedure is commonly used in computational physics to avoid discreteness artefacts [21,22].

127 The above expression is equivalently represented as a discrete convolution operation

$$
E_{i}^{a v s}=\sum_{-\infty<j<\infty} f_{j} E_{i+j}^{s} .
$$

with a tent function shown on Fig. (2a), 


$$
f_{j}=\frac{1}{C} \sum_{k=|j|}^{\infty} \frac{1}{(2 k+1)^{2}} \text { for } j \neq 0 \text {, and } f_{0}=f_{1} \text {. }
$$

Keeping the terms up to $\mathrm{n}=40$ in this sum, i.e. all terms in the sum of Eq. (4) for $|j|<40$, we are left with an error of only $2 \%$ in the tail of the weight function. The value of the normalization constant $\mathrm{C}$ is obtain ed by requiring that the average coarse grained surface energy is equal to the average local surface energy, i.e. it is set up by the condition that

$$
\sum_{-\infty<j<\infty} f_{j}=1 .
$$

\subsection{Mechanics}

The elastic energy and the normal stress at the interface are determined using a lattice spring model for the solid where elements are connected by linear elastic springs. We assume that the solid deforms only elastically without internal plastic deformation, except for the irreversible dissolution events happening at the modelled interface. Stresses in the solid are determined using an over-relaxation algorithm where elements of the model are moved until a new equilibrium configuration is found. The equilibrium configuration is defined by a given relaxation threshold. The net force $\left(F_{i}\right)$ acting on an element $i$ at position $x_{i}$ is

$$
F_{i}=\sum_{(j)} \kappa\left(x_{i}-x_{j} \mid-l\right) v_{i, j}+f_{p},
$$

where the sum is over all neighbours $j, \kappa$ is a spring constant, $l$ is the equilibrium distance between elements $i$ and $j, v_{i, j}$ is a unit vector pointing from $j$ to $i$ and $f_{p}$ is an external force like the repulsion from a wall.

The elastic energy $\left(E^{e l}\right)$ is directly evaluated at a node $i$ as

$$
E^{e l}=\frac{1}{4 V} \sum_{(j)} \kappa\left(\left|x_{i}-x_{j}\right|-l\right)^{2},
$$

where $V=\sqrt{3} / 2 l^{2}$ is the volume of an elementary cell. It can also be determined from the strain tensor $\left(u_{i k}\right)$ that is calculated from the lattice spring model with the expression [23] 


$$
E^{e l}=\frac{1}{2} \lambda_{1}\left(\sum_{i} u_{i i}\right)^{2}+\lambda_{2} \sum_{i, k}\left(u_{i k}\right)^{2},
$$

where $\lambda_{1}$ and $\lambda_{2}$ are the Lamé constants. We use the Einstein convention with summation over repeated indices. Differences in elastic energy in equation (9) refer to differences between a stressed and a non-stressed element. The Lamé constants are set up by the spring constant, the lattice constant and the hexagonal lattice configuration, i.e. $\lambda_{1}=\sqrt{3} \kappa /(2 l)$ and $\lambda_{2}=\sqrt{3} \kappa /(4 l)$, or equivalently, the Young modulus is $K=\lambda_{1}+2 \lambda_{2} / 3=2 \kappa /(\sqrt{3} l)$, and the Poisson ratio is $v=\lambda_{1} /\left(2 \lambda_{1}+2 \lambda_{2}\right)=1 / 3$ [24].

Finally the normal stress at the interface is determined from the repulsion of the two solids at the interface where the repulsion only contains normal components, assuming that a fluid film with no shear stress exists at the interface [25]. In order to calculate changes in normal stress along the interface we calculate an average of the normal stress across the whole interface and define differences in stress as the local normal stress minus the average normal stress. The simulation flow is as follows:

- First the outer walls are moved in a given time step to stress the system.

- Once the upper and lower solids meet at the interface stress builds up. The rate law (eq. 1) is then used to calculate if elements can dissolve in the given time step. If not the system is stressed again until the first element can dissolve.

- Once elements dissolve they are removed one at a time and the stress is calculated again. The time that is used up by the dissolution of a single element is averaged to be the time it takes to dissolve that element completely divided by the system size (number of elements in the x-direction). Dissolution of elements proceeds until the given time is used up and another deformation step is applied. Using a desktop workstation, each simulation lasts between 10 and 30 days; the stress relaxation being the most time consuming part. 


\subsection{Simulation parameters and disorder}

The parameters used in the simulation should mimic those of the natural example (fig. 1). For our idealized model we use a rock mainly made up of calcite with a molecular volume of $0.00004 \mathrm{~m}^{3} / \mathrm{mol}$, a Young's Modulus of $80 \mathrm{GPa}$, a Poisson's ration of 0.33 (given by the model configuration), a surface free energy of $0.27 \mathrm{~J} / \mathrm{m}^{2}$, a temperature of $300 \mathrm{~K}$ and a dissolution rate constant of $0.0001 \mathrm{~mol} /\left(\mathrm{m}^{2} \mathrm{~s}\right)$ [8]. In addition, the displacement rate of the upper and lower boundaries is fixed at a constant value corresponding to strain rates of compaction between $10^{-10}$ and $10^{-12} \mathrm{~s}^{-1}$ (see fig. 2).

In order to introduce heterogeneities to the system a bimodal variation is set on the dissolution rate constants of the elements. The heterogeneity in the system is set such that $5 \%$ of all elements have a dissolution rate constant that is half the rate constant of the other elements, i.e. they dissolve slower and pin the surface. The initial spatial distribution of the rate constants is set using a pseudorandom routine resulting in a probability of $5 \%$ of particles dissolving more slowly, picked independently for each site. Using this procedure, a spatial heterogeneity, also called quenched noise, is introduced in the initial rock.

\section{Results}

\subsection{Simulated stylolites and comparison with natural data}

At first we compare a simulated stylolite directly with a natural example. Figure 3 shows a simulation of a roughening stylolite in a model that is 400 elements wide and that has an absolute horizontal size of $40 \mathrm{~cm}$. One element in the model then has a diameter of $1 \mathrm{~mm}$ and may represent a single grain in a natural rock. We can compare the simulation with the natural stylolite shown in figure 1, especially with the inset. The simulated stylolite has a width that is about $2 / 3$ of the width of the natural stylolite in the inset (see the hammer for the scale). 
The simulated stylolite develops in 8000 years at a compaction rate of $3 \times 10^{-12} \mathrm{~s}^{-1}$ and has compacted by $25.6 \mathrm{~cm}$ or $64 \%$ (original height of the simulated box was $40 \mathrm{~cm}$ ). Both simulated and natural stylolites are visually very similar. They both produce pronounced teeth with smaller scale roughness in between and on top of the teeth. The height of the teeth (up to about $8 \mathrm{~cm}$ ) and their wid th are comparable in the natural and the simulated stylolite implying that both have similar scaling properties. The grain size of the simulated example $(1 \mathrm{~mm})$ is larger compared to natural rocks, mainly because we are limited by the calculation time of the model.

\subsection{Initiation of the stylolite roughness by interface pinning along heterogeneities}

The stylolite roughness is initiated by elements that dissolve slower. If the model contains no heterogeneity the interface will only become rough on the scale of single elements and remain flat on the larger scale. This is because surface energies and elastic energies are minimized when the surface is flat. Therefore, both of these energies will prevent the surface to roughen and the stylolite to grow [8]. Once the system contains heterogeneity, slower dissolving elements continuously pin the surface and thus make it rougher (Fig. 4).

Dissolution takes a longer time to destruct a roughness that is pinned by slower dissolving elements than to flatten an interface with no variation. The roughness is not stable but very dynamic in time since an increasing amount of more slowly dissolving elements are pinning the interface while the solid progressively dissolves. However, the slower dissolving elements themselves may dissolve as well if the roughness produces locally very high surface energies due to a strong curvature of the interface or high elastic energies due to stress concentrations or if two slower dissolving elements meet on opposing interfaces. Dissolution of pinning elements will then reduce the roughness again.

An example of the development of the roughness in a simulation and the effects of interface pinning is illustrated in figure 4. Figure 4a shows the initial random distribution of slower dissolving elements (small dark spots). This heterogeneity is frozen in to the system at 
the beginning and does not change during a simulation (quenched noise). The interface where the stylolite is initiated is shown as a black line. While the solid dissolves, pinning elements are progressively being collected within the interface (small white spots, fig. $4 \mathrm{~b}$ to d).

Two distinctively different patterns develop during the pinning of the interface. On one hand, one or a couple of elements pin very locally and produce local spikes. On the other hand, larger parts of the interface may be pinned between two elements that are further apart. These second pattern generates the teeth geometries, typical of stylolites, with teeth having various widths.

Figure $4 \mathrm{e}$ illustrates three different cases of pinning schematically. Single pinning elements produce spikes whereas two pinning elements that pin from the same side produce teeth. The structures grow fastest when elements pin from opposing sides. The interfaces in figures $4 \mathrm{c}$ and $4 \mathrm{~d}$ illustrate the teeth-forming processes presented in figure $4 \mathrm{e}$ : the interface is made up of single pinning elements, larger teeth where groups of elements pin and steep interfaces where elements pin from opposing sides. The surface structure changes when new pinning elements are collected with in the stylolite and when pinning elements are destructed. The amplitude of the stylolite grows during these processes (from figure $4 \mathrm{~b}$ to $\mathrm{d}$ ) and the wavelength of the interface is also evolving. Small wavelengths can grow very fast (figure 4c) whereas the larger wavelengths need longer time to develop (figure 4d).

Figure 5 shows the evolution of the growing roughness of two stylolites through time in 3D diagrams where the $\mathrm{x}$-axis shows the amount of elements in the $\mathrm{x}$ direction, the $\mathrm{y}$-axis corresponds to the time in model time-steps (here one step corresponds to 20 years) and the $z$ axis shows the non-dimensional height of the stylo lite. The parameters for the two stylolites shown in figure $5 \mathrm{a}$ and $5 \mathrm{~b}$ are the same except for the absolute length (and height respectively). The stylolite shown in figure $5 \mathrm{a}$ has an $\mathrm{x}$-dimension of $0.4 \mathrm{~cm}$ whereas the stylolite shown in figure $5 \mathrm{~b}$ has an $\mathrm{x}$-dimension of $40 \mathrm{~cm}$. 
The differences in abso lute initial system size $L$ have an effect on the dominance of elastic versus surface energies during the roughening process. Surface energies become increasingly more important towards smaller scales. This means that the stylolite in figure $5 \mathrm{a}$, which is relatively small $(L=0.4 \mathrm{~cm})$ with a small grain size of $10 \mu \mathrm{m}$, is dominated by surface energies so that elastic energies only play a minor role. The stylolite shown in figure $5 \mathrm{~b}$ however is relatively large $(L=40 \mathrm{~cm})$ with a large grain size of $1 \mathrm{~mm}$ so that the elastic energy dominates the roughening process and surface energies only play a minor role. When comparing figures $5 \mathrm{a}$ and $\mathrm{b}$ (note that the z-axis scales differently, one unit corresponds to $0.4 \mathrm{~cm}$ in a) and to $40 \mathrm{~cm}$ in b)), one observes that the roughness forms better developed teeth with steep sides in the case of the larger stylolite (fig. 5b). The roughness of the smaller stylolite (fig. 5a) is not growing smoothly but is disrupted quite often and produces neither large amplitude nor well-developed teeth. In addition, the larger stylolite (Fig. 5b) grows progressively while the small stylolite (Fig. 5a) shows an initial increase in roughness that is followed by strong fluctuations in time, where the average roughness amplitude remains more or less constant. These roughness evolutions imply that pinning elements are destroyed when surface energy is high because of very high curvatures of spikes. The interface of the surface energy dominated stylolite therefore contains no larger spikes or teeth and is quite dynamic. Elastic energy on the other hand does not destroy spikes easily. Therefore well-developed teeth structures tend to arise in larger stylolites at the outcrop scale when surface energy is relatively unimportant, whereas we would expect to find less welldeveloped teeth but rounder structures on the scale of a thin-section, where surface energy is important.

In order to explore the evolution of the roughness amplitude with time, we use signalprocessing tools from statistical physics [26], as illustrated in the next section.

\subsection{Growth of the roughness with time}


280 laws that are the same or at least very similar for different interfaces and surfaces (as e.g., gas-

281 fluid interface motion in non-saturated porous media, propagation of flame fronts, atomic

282 deposition processes, bacterial growth, erosion or dissolution fronts, contact line motion

283 biphasic fronts over disordered pinning substrates, interfacial crack fronts). These laws,

284 discovered by statistical physicists, describe how the amplitude of the roughness grows non-

285 linearly with time, following power laws [26-29].

286 First we have to define an average value for the amplitude of the roughness of our

287 numerical stylolites for each time step. We use the root mean square method to determine the

288 average width of the stylolite roughness following [26]

$$
w(L, t) \equiv \sqrt{\frac{1}{L} \sum_{i=1}^{L}[h(i, t)-\bar{h}(t)]^{2}},
$$

where $w$ is the interface width as a function of system size $L$ and time $t, h$ is the height of characterizes the growth of the roughness. In our simulations, the system size $L$ is defined as the number of elements in the x-direction. We use model sizes of 200 and 400 elemen ts in the $\mathrm{x}$-direction. The average height of the interface is defined as interfacial systems, roughening interfaces grow following a power law in time with a socalled growth exponent $\beta$. This is described by

$$
\bar{h}(t) \equiv \frac{1}{L} \sum_{i=1}^{L} h(i, t)
$$

301 where the interface width $w$ is proportional to time $t$ to the power $\beta$, for a given system size $L$. If $\beta=1.0$, the interface grows linearly with time, if $\beta$ is smaller than 1.0 the interface growth 
slows down with time. Normal diffusion processes are characterized by $\beta=1 / 2$, anomalous diffusion processes by $\beta \neq 1 / 2$, and $0 \leq \beta \leq 1$. In addition, for most stochastic interfacial systems the width of roughening interfaces saturates after a critical time $t_{c r i t}$. This time increases with the system size $L$.

When modelling the growth of stylolites, one can expect two scaling regimes in time, first a power law up to time $t_{\text {crit }}$ followed by a regime where $w$ remains constant (Fig. 6a). In order to study the dynamics of the roughening process one constructs diagrams of $\log _{10}(w)$ against $\log _{10}(t)$. The increase in wid th of the interface roughness should follow a straight line where the slope of the line gives the growth exponent $\beta$. After a critical time $t_{c r i t}$ the roughness saturates and the slope vanishes to zero (Fig. 6a).

We studied three simulations (Fig. 6b-d) with this method, where the simulation shown in figure $6 \mathrm{~b}$ has a system size $L=0.4 \mathrm{~cm}$, the simulation shown in figure $6 \mathrm{c}$ has $L=4$ $\mathrm{cm}$ and the one shown in figure $6 \mathrm{~d}$ has $L=40 \mathrm{~cm}$. Figure $6 \mathrm{~b}$ and $5 \mathrm{a}$ and figure $6 \mathrm{~d}$ and $5 \mathrm{~b}$ show the same simulations, respectively. The simulated stylolite shown in figure $6 \mathrm{~b}$ shows the expected behaviour with two scaling regimes, the roughness first grows with a roughness exponent of 0.5 and saturates after 2500 years, where it remains constant close to a value of $50 \mu \mathrm{m}$. Go ing back to figure $5 \mathrm{a}$ where the growth of the same stylolite is illustrated in $3 \mathrm{~d}$, the roughness saturates after 2500 years, which corresponds to model time step 125 . Figures $6 \mathrm{c}$ and d show only the first scaling regime where the increase in roughness amp litude $w$ follows a power law, but $w$ never saturate. That means probably that these two simulations (Fig. 6c, d) did not reach the critical time needed for the roughness to saturate. Taking a look at figure $5 \mathrm{~b}$ where the growing roughness of the stylolite shown in figure $6 \mathrm{~d}$ is illustrated, the roughness width still grows i.e. is not yet saturated.

The three stylolites shown in figure $6 b-d$ seem to have different critical times when the roughness saturates but also their growth exponents vary. $\beta$ increases from the smallest simulation (fig. $6 \mathrm{~b}$ ) with a value of 0.5 through the medium-sized simulation with a value of 
0.54 to the largest simulation with a value of 0.8 . These differences may reflect differences of growth regimes that are dominated by either surface energy or by elastic energy, in analogy to the discussion on figures $5 \mathrm{a}$ and $\mathrm{b}$. The surface energy dominated growth regime (small stylolite, fig. 6b) has a growth exponent of 0.5 and saturates relatively early. The stylolite shown in figure $6 \mathrm{c}$ is intermediate, the growth exponent is still small with 0.54 but the roughness does not saturate with in the simulated time. The larges stylolite (fig. 6d) has a significantly larger growth exponent of 0.8 , does not saturate, and represents the elastic energy dominated regime.

In terms of natural stylolites the above-mentioned values demonstrate that small stylolites that grow with in the surface energy dominated regime grow as slow as a diffusive process (exponent of 0.5 ) and saturate early so that compaction estimates are almost impossible. However, stylolites that grow within the elastic energy dominated regime grow faster (exponent 0.8 ) and do not seem to saturate after 8000 years. They can thus capture part of the compaction of the rock even though their growth is non-linear, and slows down with time. Consequently, rather than using a rule of thumb as a direct proportionality between $A$, the amplitude of compaction displacement accommodated for around a stylolite, and the stylolite amplitude $w$, the nonlinear power-law observed for this process where the imposed displacement is linear in time can be utilized to be stated as $(w / l) \sim\left(t / t_{0}\right)^{\beta} \sim(A / l)^{\beta}$, with $l$ the relevant physical len gth, which is here the grain size.

Conversely, for large enough stylolites, as long as the critical saturation time has not been reached at the observed scale, the relationship between the total compaction displacement $\mathrm{A}$ and the stylolite amplitude should be of the type

$$
A \sim(w / l)^{1 / \beta} l .
$$

This law should hold until the critical saturation time is reached. Using the proposed scaling law for the large simulation $(L=40 \mathrm{~cm})$ we obtain the right relation with a slope of 6.6 (Fig. 7) using the root mean square width and a grain size of $1 \mathrm{~mm}$. The stylolite amplitude may 
finally saturate, but this seems not to happen in 8000 years for the case of the $40 \mathrm{~cm}$ long stylolites that we have studied.

3.4 The teeth structures and their relation with the main compressive stress direction

After characterizing the dynamics of the growth process, we now focus on the orientation of the stylolite teeth. We have demonstrated so far that the teeth mainly develop in the regime where elastic energy is dominant so that well-developed teeth form in the stylolite simulation with a system size $L=40 \mathrm{~cm}$. The steep sides of the teeth are thought to develop parallel to the main compressive stress and the top of the teeth is thought to be oriented perpendicularly to that stress direction.

We test this hypothesis that the teeth track the orientation of the largest principal stress. For the test, we choose again a model with an initially flat dissolution surface that is or iented horizontally with respect to the simulation box but we rotate the compression direction so that the stress field rotates. Theoretically, the teeth should follow the rotated stress field so that one can evaluate the direction of the main compressive stress using the teeth orientation. We use simulations with a system size of 200 elements in the $\mathrm{x}$-direction with an absolute size of $L=10 \mathrm{~cm}$ so that we are in the elastic regime.

The results of the 5 different simulations are shown in figure 8 a-e where the orientation of the compression direction is shown on the left hand side. Indeed, the teeth do follow the stress directions in all the examples. The sides of the teeth are the best stress direction indicators but even the tops of some teeth tend to orient themselves with respect to the stress. The most extreme example is shown in figure $8 \mathrm{e}$ where the compression direction has a very low angle with respect to the initial horizon tal heterogeneity. The initially horizontal surface has vanished and a stair-step geometry develops with steps that are oriented perpendicular with respect to each other. One set of surfaces corresponds to the sides of the teeth and the other set to the top surface of the teeth. 

main compressive stress and can therefore be used as stress indicators in natural rocks.

\subsection{Model for the determin istic orientation of stylolite teeth}

Our simulations have shown that well-developed stylolite teeth only tend to develop in

384

the elastic regime (Fig. 5). In order to understand the relation between the compaction direction and the orientation of the stylolite teeth, we consider the stress distribution at the interface and the finite compaction. The stress field at the interface for a given time step is directly controlling dissolution. However, just observing the stress field across a stylolite for a single time step is not enough since dissolution and thus a change in the geometry of the interface influences the stress back, dissolution and stress being highly coupled. Therefore we also have to consider the whole stress history and the finite compaction across the interface. Going back to figure 4, one can observe that the location of the teeth is random because it depends on the quenched noise in the background. The second simple observation is that the finite compaction is directly recorded by the pinning of particles (Fig. 4). As long as they pin the interface, they move in the direction of the compaction, which is parallel to the far field compressive stress for a simple homogeneous rock. This scenario does not change when the compaction direction and thus the main compressive stress is not perpendicular to the initial interface (Fig. 9a). Pinning particles still record the rotated compaction direction because they move in that direction. If two pinning elements are close but on opposing sides of the interface they move in opposite directions and a perfect side of a tooth develops recording the compaction direction. If this direction is parallel to the far field main compressive stress, the stylolite also records this stress direction.

Looking at the movement of a whole interface (Fig. 9b), one can see that the total amount that the interface may move perpendicularly to itself depends on its orientation with respect to the compaction direction. An interface that is oriented perpendicularly to the compaction direction can have the largest fluctuations and thus produce a roughness with the largest 
amplitude. An interface that is oriented at a smaller angle to the compaction direction will not develop importantly in the direction perpendicular to itself. If particles are pinning this interface, it may actually develop steps. The most extreme case is an interface that is oriented parallel to the compaction direction. This interface shows no fluctuations and thus cannot develop a roughness. It cannot even develop steps because particles cannot pin this interface. The interface is very stable and can only act as a transform fault. Therefore the sides of teeth, which reflect such an interface, are very stable and the teeth geometry itself is a natural consequence of the compaction direction (Fig. 9c). Strongly pinning particles are not even necessary for the development of these geometries. Some pinning or a variation in dissolution across the interface is necessary for a roughening of the interface, but once this roughness is initiated and for large scales where the surface energy is not dominant, the typical teeth geometries develop and stay stable without much flickering, i.e. they are strongly deterministic.

Looking at the stress history, we also show that the main compressive stress close to the stylolite is parallel to the main compaction direction. An interface perpendicular to the main compaction direction has a significantly normal stress component and experiences dissolution. For an interface parallel to the main compressive stress, dissolution will only relax the second principle stress that builds up in a laterally confined system. The second principle stress will vanish to zero and in the extreme case a hole can develop. Compaction itself cannot fill the hole because the compaction has the wrong direction. The only possibility to build-up stress again is a flow of material into the hole or a collapse of the teeth. Looking at the stress history, the main compressive stress close to the stylolite and the direction of the finite compaction are parallel. Indeed, the stylolite teeth track this direction.

\section{Discussion}

In this discussion we first focus on several as sumptions used in the simulations. The focus of these simulations is the roughen ing process of an initially smooth interface, not an 
investigation of the reason why the dissolution is localized. Therefore we use an initially flat

433 interface were dissolution starts and restrict dissolution to this stylo lite surface. Some

434 observations in natural rocks support such a scenario: First, some stylolites do initiate from mica-rich layers. Next, some stylolites with oblique teeth do exist. Stylolites with oblique teeth are most probably initiating from an interface that was oblique with respect to the main compressive stress direction when the stylolite roughness started to grow. However, surely not all stylolites start from given heterogeneities but may localize due to geochemical selforganization [30] or anti-cracking [16]. This is beyond the focus of this current manuscript. In our simulations, we use a simple description of the noise that initiates the roughening process. As mentioned earlier, the no ise is only chemical (a variation in dissolution constants), is set on single particles that have the same size and is distributed with a bimodal distribution. In a real rock, elastic properties and surface energy may also vary, the noise may be on the grain scale or at the scale of smaller heterogeneities and the distribution may be more complicated than bimodal. It is not clear how variations in these parameters may affect the stylolite growth and what kind of noise is present in a real rock. The nature of the noise may influence pinning of the surface and thus may influence the growth and structure of the stylolite [31]. However, since our simulations can reproduce natural stylolites quite realistically, one can argue that the exact nature of the noise is less important than the effects of elastic energy, surface energy and stress.

Another interesting question is how a stylolite grows when the finite strain is rotating. We have demonstrated that stylolite teeth develop by a particle pinning process at the interface and track the direction of the finite compaction. However, this is not necessarily true if the finite strain is rotating because particles at the interface only pin the interface from the moment when they meet the interface to the moment when they dissolve. This means that they are only recording parts of the finite compaction direction. If the stylolite grows in a simple shear dominated rock, the direction of the pinning particles should initially record the 
direction of the compressive ISA (incremental stretching axes) and then the teeth should rotate. Therefore young teeth do really track the compressive ISA and thus probably also the direction of the compressive stress whereas old teeth rotate. However, if the roughness is dynamic and the stylolite constantly changes its shape, it can always grow new teeth and old teeth will disappear so that the direction of the compressive ISA and the compressive stress are recorded.

\section{Conclusions}

We have developed a numerical model that can successfully reproduce the roughening of stylo lites. The numerical stylolites are very similar, if not identical, to natural stylolites. We propose that the growth of the stylolite roughness is induced by pinning particles, that produce a complex interface that evolves dynamically through time. Two different regimes can be separated, a small-scale regime where the roughness fluctuates significantly and a large-scale regime where well-developed teeth pattern s grow. The small-scale regime is dominated by surface energy whereas the large-scale regime is dominated by elastic energy. Scaling laws characterizing the dynamic growth of the stylolite roughness as a function of time are proposed. These laws show that the roughness grows in two successive regimes in time, a first regime where the growth follows a power law and a second regime where the roughness growth saturates. These findings are essential for compaction estimates using stylolites, the roughness growth is non-linear in time, slows down with time and may even saturate. A saturated stylolite looses its memory for compaction completely and cannot be used for total compaction or strain rate estimates. We also show with our simulations that the teeth of stylolites do really follow the main compaction direction and may thus indicate the direction of the maximum compressive stress in a homogeneous rock. We show that this strong deterministic geometry of the teeth is a consequence of pinning particles that move in the direction of the finite compaction and of the local stress history at the stylolite interface. Summarizing, the geometry of stylolite teeth can be used by geologists to estimate the 
direction of the finite compaction or the main compressive stress, but absolute compaction estimates are difficult to perform and may strongly underestimate the real values. In the regime preced ing saturation, we utilize the observed nonlinear growth of the roughness amplitude to propose a refined estimate of absolute compaction, Eq. (13), based both on the stylolite roughn ess amplitude and the size of the dissolving grains (Fig. 7). This can in general be used as a lower bound of total compaction.

\section{Acknowledgements:}

DK acknowledges funding by the MWFZ Center of Mainz University and the DFG (KO 21 14/5-1). This is contribution No. 37 from the Geocycles Cluster funded by the state of Rhein land-Pfalz.

\section{References}

[1] P.B. Stockdale, Stylolites: their nature and origin. Indiana University Studies IX, (1922) 197.

[2] M.T. Heald, Stylolites in sandstones. The Journal of Geology. 63, (1955) 101-114.

[3] W. Park, E. Schot, Stylolites: their nature and origin, J. Sedimen. Petrol., 38, (1968) 175191.

[4] R. Bathurst, Carbonate sediments and their diagenesis, Elsevier Sci. New York, (1971).

[5] B. Bayly, A mechanism for the development of stylolites, Journal of Geology, 94, (1986) 431-435.

[6] F. Renard, J. Schmittbuhl, J.-P. Gratier, P. Meakin, E. Merino, Three-dimensional roughness of stylolites in limestones, J. Geophys. Res., 108, (2004) B03209.

[7] Z. Karcz, C.H. Scholz, The fractal geometry of some stylolites from the Calcare Massiccio Formation, Italy. Journal of Structural Geology, 25, (2003) 1301-1316. 
[8] J. Schmittbuhl, F. Renard, J.-P. Gratier, R. Toussaint, The roughness of stylolites:

Implications of 3D high resolution topography measurements, Phys. Rev. Lett., 93, (2004) 238501.

[9] J. Suppe, Principles of Structural Geology, Prentice-Hall, New Jersey, (1985) 537.

[10] H. Stel, M.J. De Ruig, Opposite vergence of a kink fold and pressure solution cleavage, southeast Spain: a study of the relation between paleostress and fold kinematics. Tectonophysics 165, (1989) 117-124.

[11] J.-P. Petit, M. Mattauer, Plaeostress superimposition deduced from mesoscale structures in limestone: the Matelles exposure, Languedoc, France. Journal of Structural Geology 17, (1995) 245-256.

[12] M. Ebner, B. Grasemann, Divergent and convergent non-isochoric deformation. Journal of Structural Geology, 28, (2006)1725-1733.

[13] D.C.P. Peacock, I.N. Azzam, Development and scaling relationships of a stylolite population. Journal of Structural Geology, 28, (2006) 1883-1889.

[14] J. Gratier, L. Muquet, R. Hassani, F. Renard, Experimental microsylolites in quartz and modeled application to natural stylolitic structures, Journal of Structural Geology, 27, (2005) 89-100.

[15] C.N. Drummond, D.N. Sexton, Fractal structure of stylolites, Journal of Sedimentary Research, 68,(1998) 8-10.

[16] R.A. Fletcher, D.D. Pollard, Anticrack model for pressure solution surfaces. Geology 9, (1981) 419-424.

[17] R. Katsman, E. Aharonov, H. Scher, Localized compaction in rocks: Eshelby's inclusion and the spring network model. Geophysical Research Letters, 33, (2006) L10311

[18] M.W. Jessell, P.D. Bons, L. Evans, T.D. Barr, K. Stüwe, Elle, The numerical simulation of metamorphic and deformation textures. Computers and Geosciences, 27, (2001) 17-30. 
[19] D. Koehn, J. Arnold, A. Malthe-Sørrenssen, B. Jamtveit, Instabilities in stress corrosion 535 and the transition to brittle failure, American Journal of Science, 303, (2003) 956-971.

536 [20] D. Koehn, A. Malthe-Sørenssen, C. Passchier, The structure of reactive grain-bound aries 537 under stress containing confined fluids. Chemical Geology, 230, (2006) 207-219.

538 [21] S. McNamara, E.G. Flekkoy, K.J. Maløy, Grains and gas flow: Molecular dynamics with 539 hydrodynamic interactions. Phys. Rev. E 61, (2000) 4054-4059.

540 [22] O. Johnsen, R. Toussaint, K.J. Maløy, E.G. Flekkøy, Pattern formation during air 541 injection in to granular materials confined in a circular Hele-Shaw cell. Phys. Rev. E, 74, $542 \quad$ (2006) 011301.

543 [23] L.D. Landau, E.M. Lifshitz, Theory of Elasticity. New York, Pergamon Press, (1959) $544 \quad 134$.

545 [24] E.G. Flekkøy, A. Malthe-Sørrensen, B. Jamtveit, Modeling hydrofracture. Journal of 546 Geophysical research B8, 107, ECV 1, (2002) 1-11.

547 [25] M.S. Paterson, Nonhydrostatic thermodynamics and its geologic applications. Reviews $548 \quad$ of Geophysics Space Physics, 11, (1973) 355-389.

549 [26] A. L. Barabási, E.H. Stanley E. H., Fractal concepts in surface grow th, Cambridge Univ $550 \quad$ Press. New York (1995).

551 [27] F. Family, T. Vicsek, Dynamics of fractal surfaces. World Scientific Publishing, $552 \quad$ Singapore New Jersey London Hong Kong (1991).

553 [28] S. Moulinet, A. Rosso, W. Krauth, E. Rolley, Width distribution of contact lines on a 554 disordered substrate, Phys. Rev. E, 69, (2004) 035103(R).

555 [29] K.J. Maløy, S. Santucci, J. Schmittbuhl, R. Toussaint, Local waiting time fluctuations 556 along a randomly pinned crack front. Phys. Rev. Lett., 96, (2006) 045501.

557 [30] T. Dewers, P. Ortoleva, A coupled reaction/transport/mechanical model for intergranular 558 pressure solution, stylolites, and differential compaction and cementation in clean 559 sandstones. Geochimica et Cosmochimica Acta, 54, (1990) 1609-1625. 
560 [31] A. Brouste, F. Renard, J.-P. Gratier, J. Schmittbuhl, Variety of stylolite morphologies 561 and statistical characterization of the amount of heterogen eities in the rock, Journal of 562 Structural Geology, in press (2007). 


\section{Figure captions}

564 Figure 1. Natural stylo lites on a limestone quarry surface from the Burgundy area, France. On the large scale the stylolites are planar structures whereas on the small scale they show a pronounced roughness (see inset). Note the steep "teeth" like patterns in the inset.

Figure 2. a) weight function used in the coarse graining procedure to evaluate the surface energy. b) setup of the numerical model. Side-walls are fixed whereas the upper and lower walls are moved inwards to compact the system. The rock contains two kinds of particles that dissolve at two different rates. This heterogen eity in the dissolution rate represents an initial quenched noise in the rock.

Figure 3. Simulated stylolite with a width of $L=40 \mathrm{~cm}(1: 1)$. Note similarities between the natural stylo lite (in Figure 1) and the simulated stylo lite. Both develop a roughness on different scales and well-developed square teeth structures.

Figure 4. Particles pinning along the interface. (a) Initial setup of a simulation with the in itial interface in the centre and the quenched noise in the background (particles that dissolve twice slower are dark). (b)-(d) Progressive growth of the roughness and pinning of the interface (particles that pin are white). (e) Structures that develop during pinning: spikes, teeth that are pinned in one direction and teeth that are pinned in two directions.

Figure 5.3D diagrams showing the growth of two stylolites. (a) Small stylolite that grows in the surface energy dominated regime ( $\mathrm{x}$ dimension is $L=0.4 \mathrm{~cm}$ ). The growth of the roughness slows down relatively fast and saturates. The growth is very dynamic so that pinning particles are dissolved relatively fast. (b) Large stylo lite that grows in the elastic 
energy dominated regime (x dimension is $L=40 \mathrm{~cm}$ ). The stylolite roughness grows continuously and develops well-developed teeth.

Figure 6. Log/log diagrams showing the growth of the roughness amplitude $w$ (one unit $=$ $0.004 \mathrm{~m}$ ) against time (t, model steps). (a) Schematic diagram illustrating the two regimes that should develop [26]. Regime I shows a growth that follows a power law in time with a growth exponent $\beta$ until a critical time. Regime II is characterized by a saturation of the growth so that the roughness amplitude remains constant. (b) Small stylolite $(L=0.4 \mathrm{~cm})$ that shows both regimes, a power law growth with an exponent of 0.5 in regime I (as slow as a diffusive process) and a crossover regime II where the roughness saturates. (c) Medium sized stylolite $(L=4 \mathrm{~cm})$ that shows only the regime I with a power law growth with an exponent of 0.54 . (d) Large stylolite $(L=40 \mathrm{~cm})$ that shows only regime I with a power law growth with an exponent of 0.8 .

Figure 7. Proposed scaling relation between the amplitude of compaction around a stylolite $(A)$ and the mean width of the stylolite roughness $(w) . l$ corresponds to the grain size and $\beta$ is the predetermined growth exponent ( 0.8 in this case). Plot shows the scaling relation for the large stylolite simulation (40 $\mathrm{cm}$ long stylo lite) using equation 13 . Theoretically this scaling relation with the determined prefactor of 6.6 can be used to estimate compaction from real stylolites.

Figure 8. Stylolite teeth directions track variations of the compression direction. The orientation of the initial interface is given at the top and the direction of the compaction is illustrated at the left hand side. The simulations show that the orientation of the teeth is strongly deterministic and follows the compaction direction. 
614 Figure 9. Model for the dev elopment of stylolite teeth. (a) Development of oblique teeth due 615 to pinning particles that record the relative movement of the rock on each side of the interface.

616 (b) Possible fluctuations that may develop on interfaces with different orientations with 617 respect to the main compaction direction. Strongest fluctuations appear on interfaces that are 618 oriented perpendicular with respect to the main compaction direction (arrows). Interfaces that 619 are parallel to the main compaction direction do not show fluctuations. (c) Illustration of these 620 fluctuations on a natural stylolite. The sides of teeth are relatively stable, show no fluctuations 621 and may act as transform faults. Plateaus of teeth are interfaces that show largest fluctuations. 


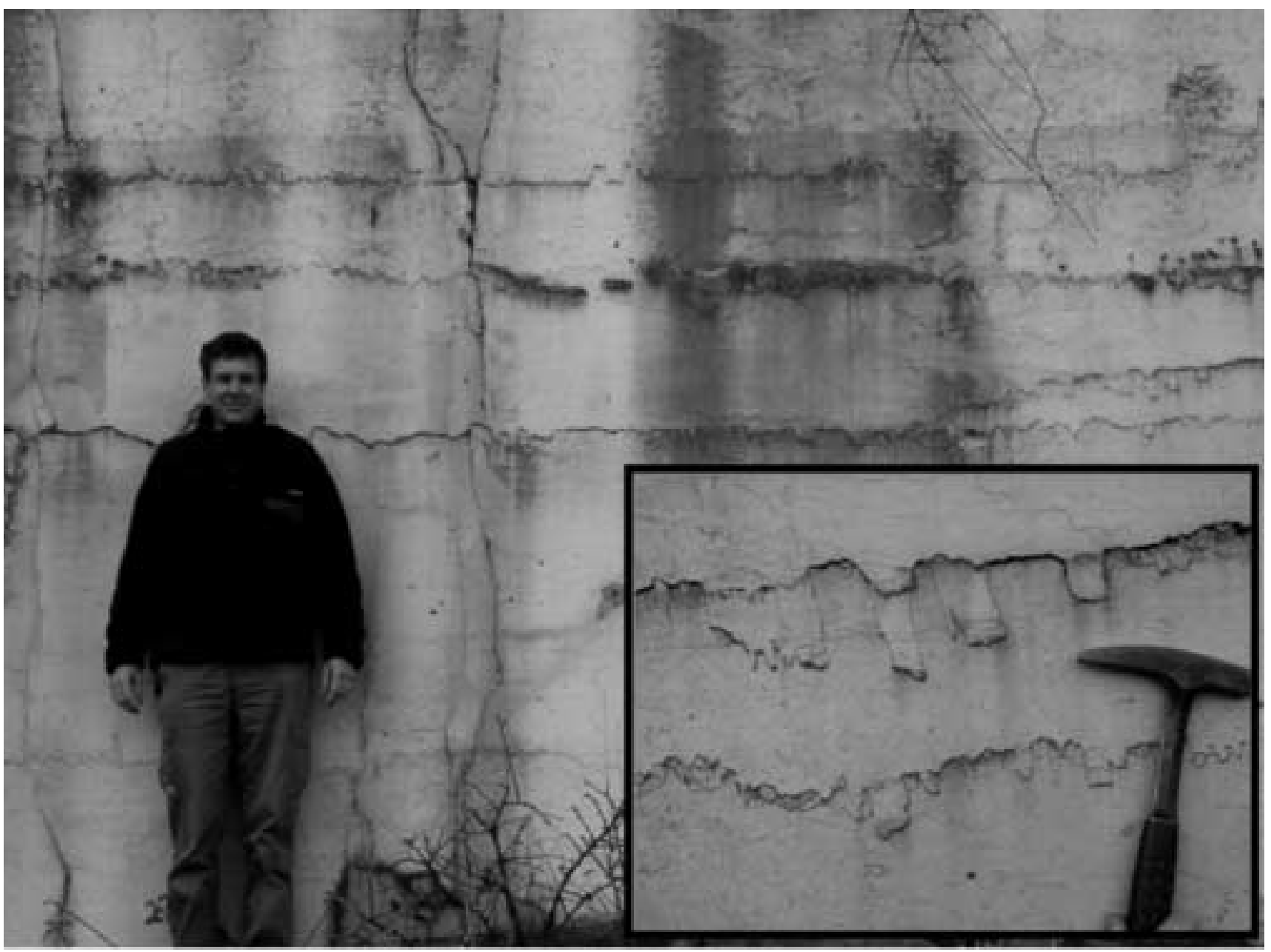

Fig. 1 
constant

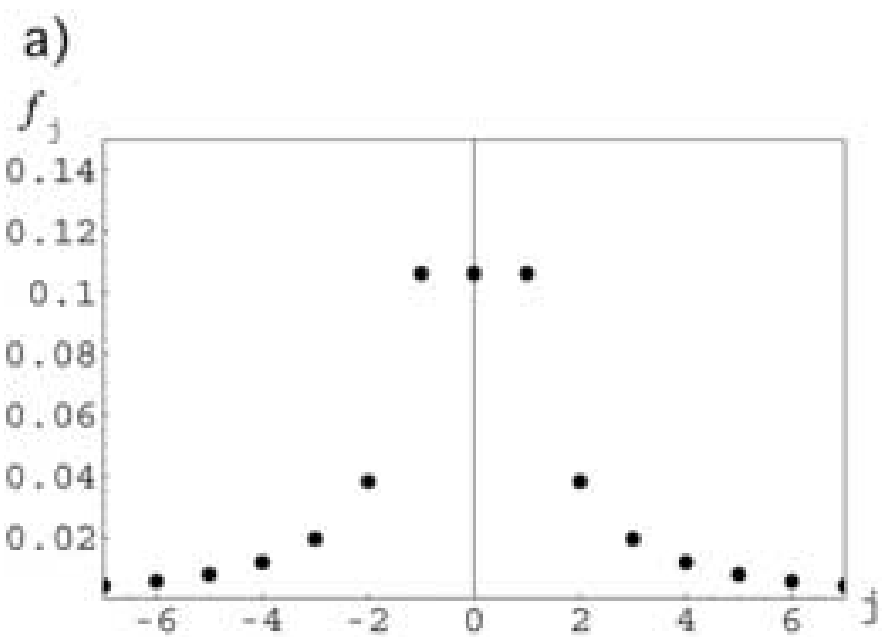

b)

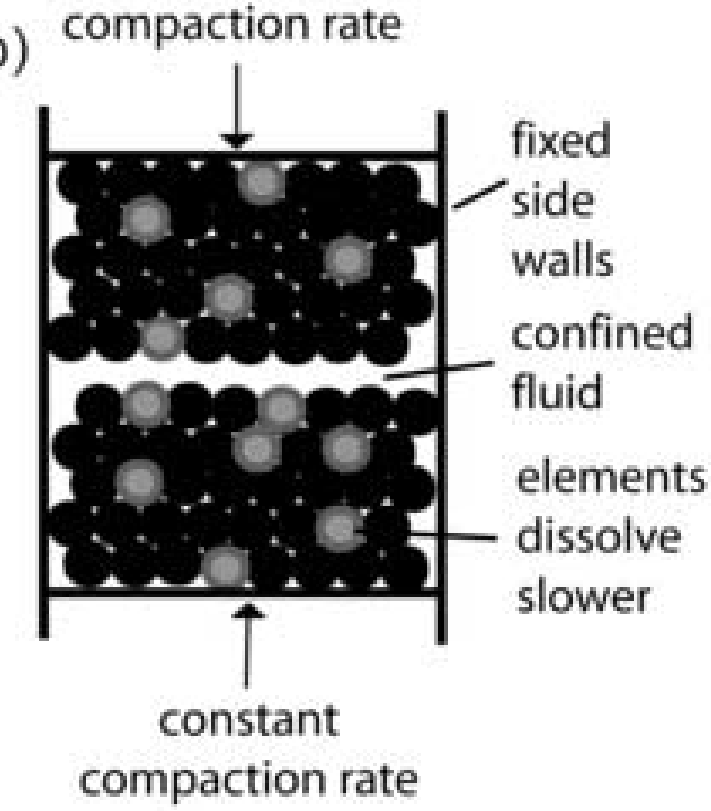

Fig. 2 


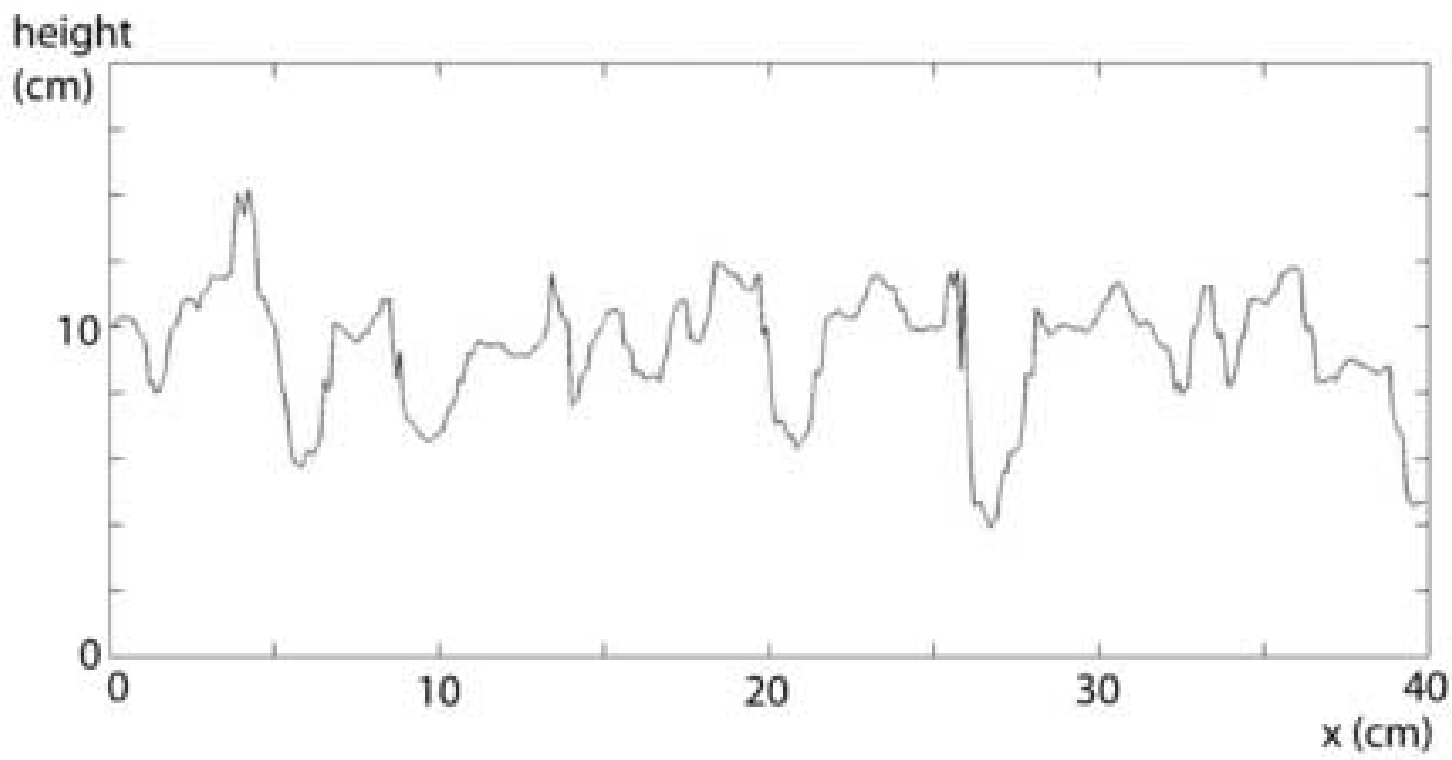

Fig. 3 
Click here to download high resolution image

(a)

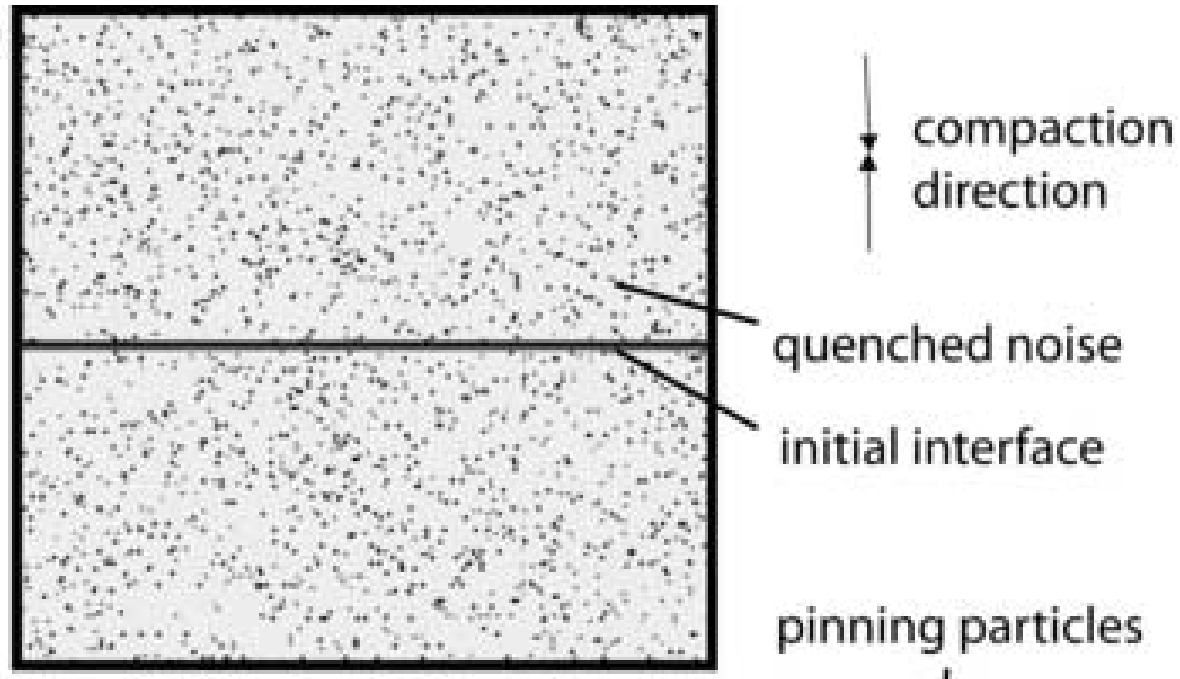

(b)

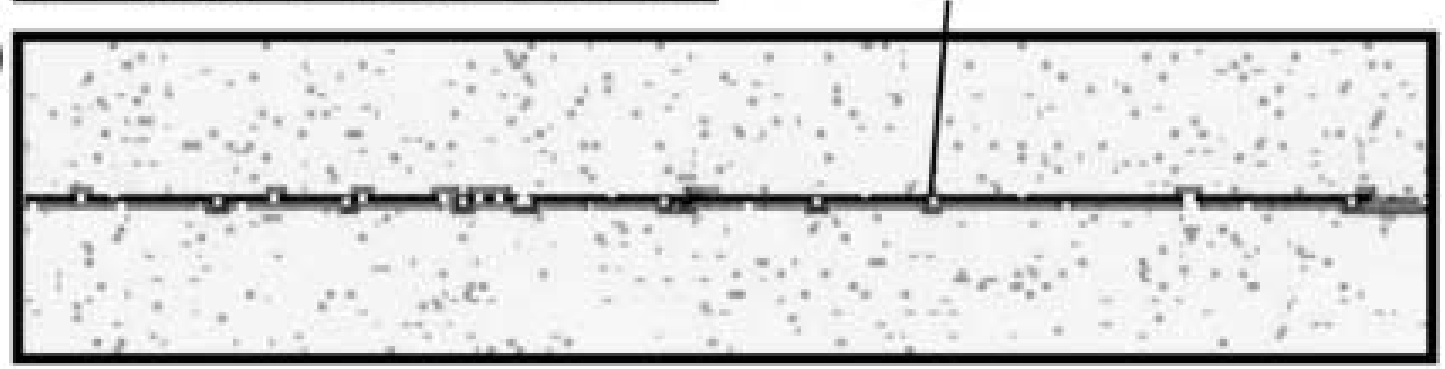

(c)

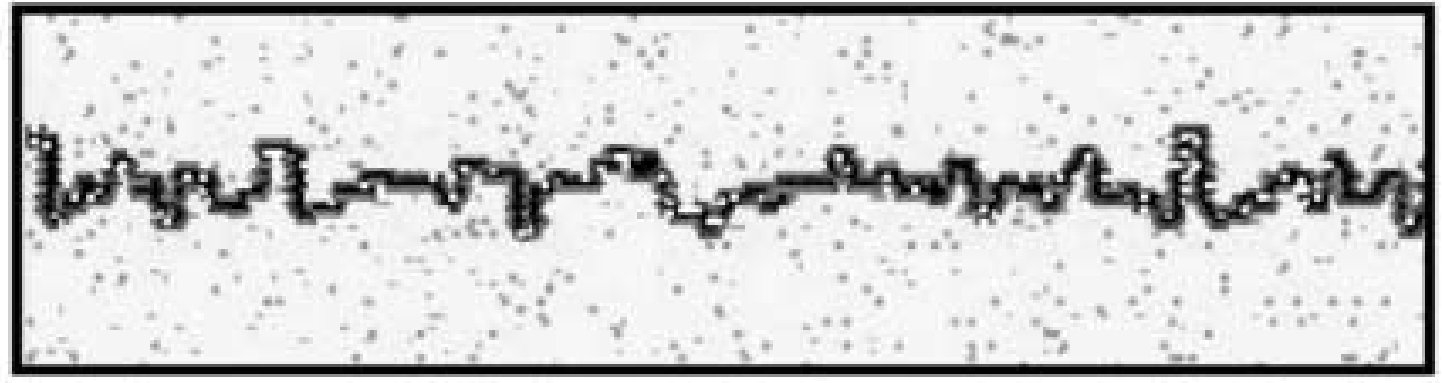

(d)

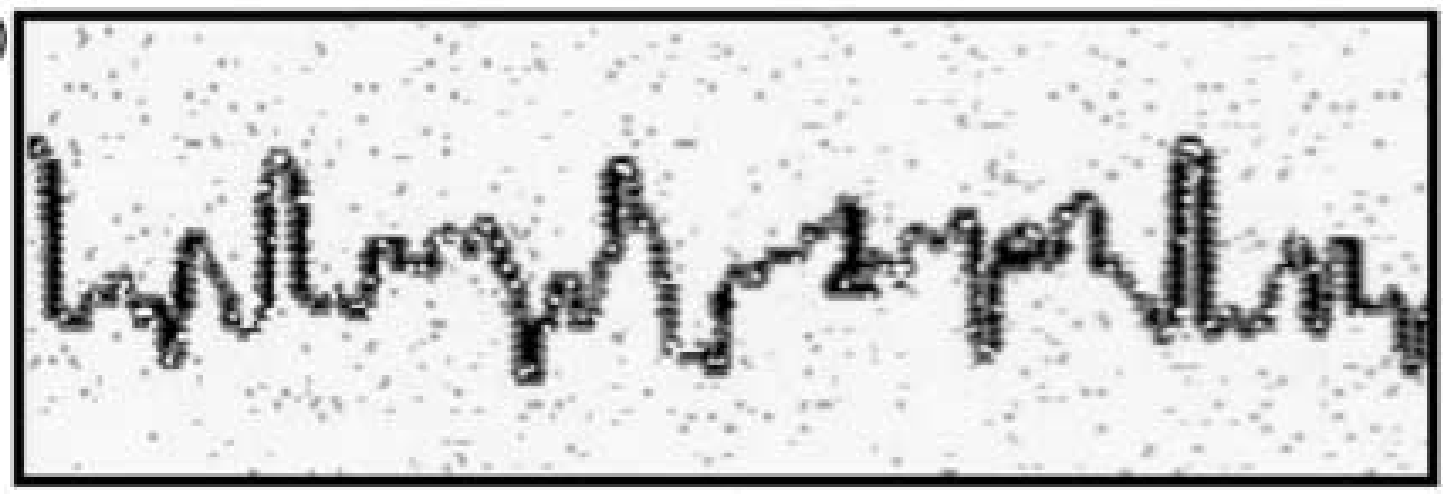

(e)

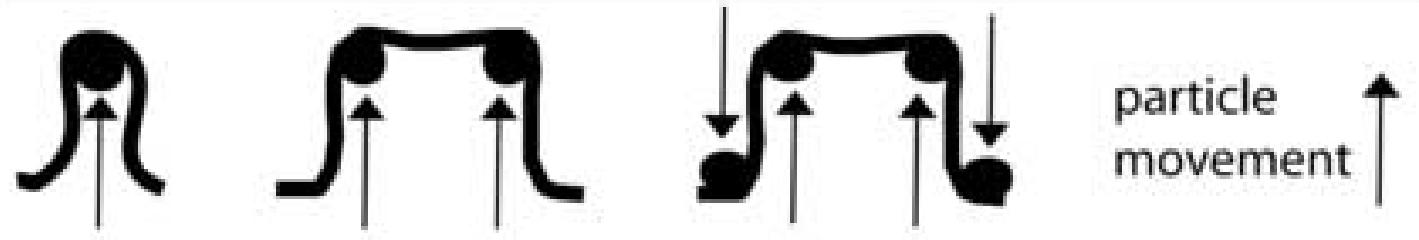

Fig. 4 
a)

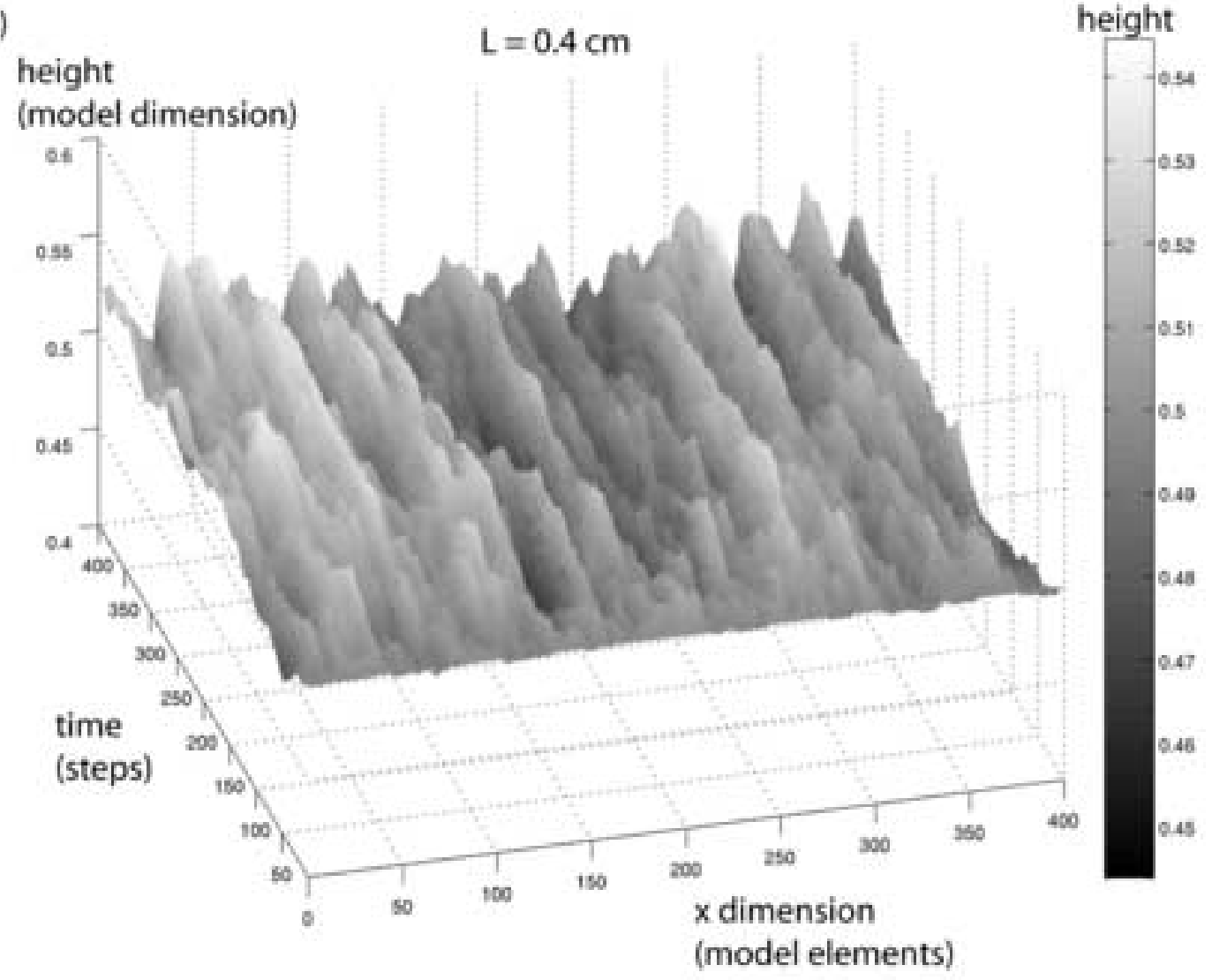

b)

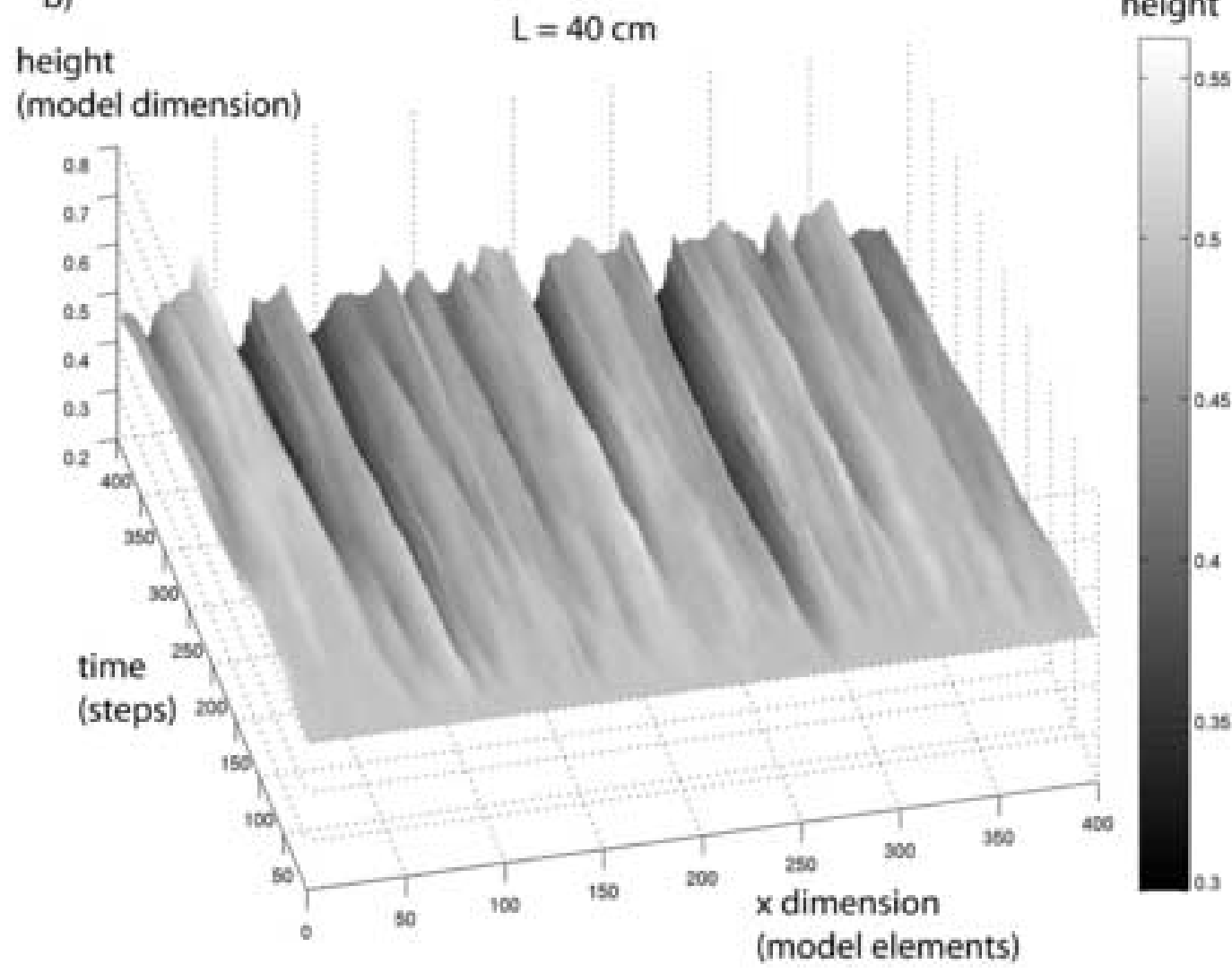

Fig. 5 
a)
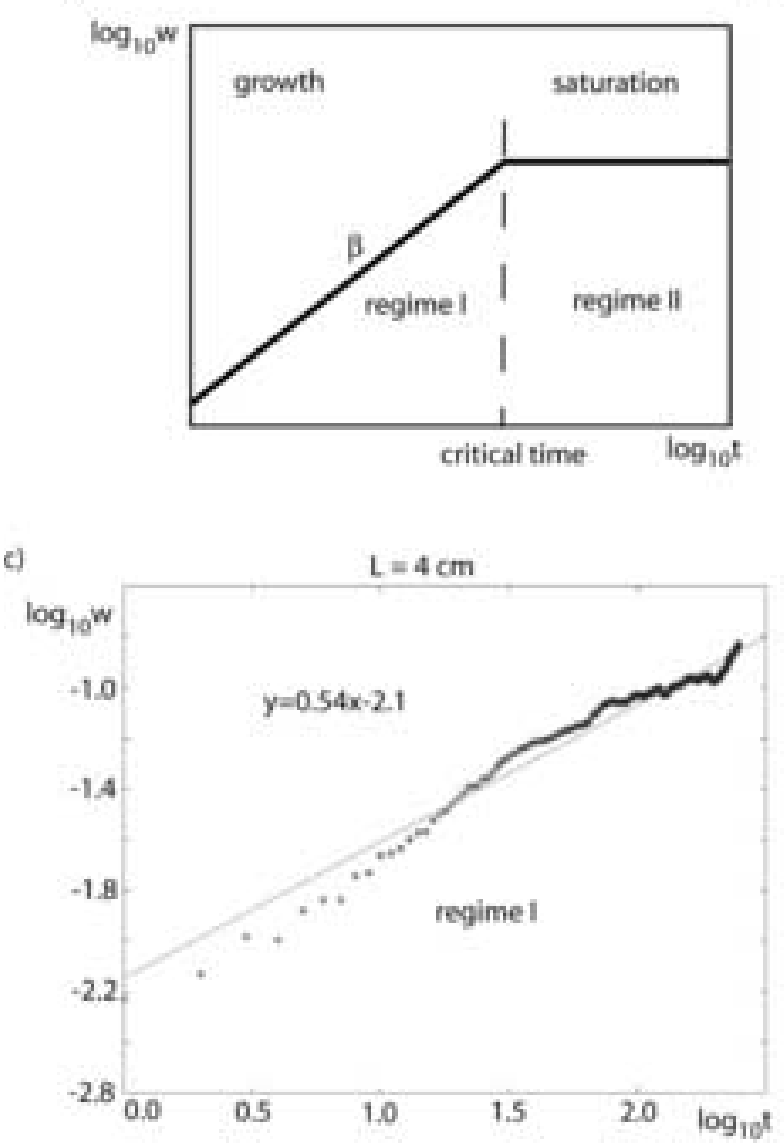

b)

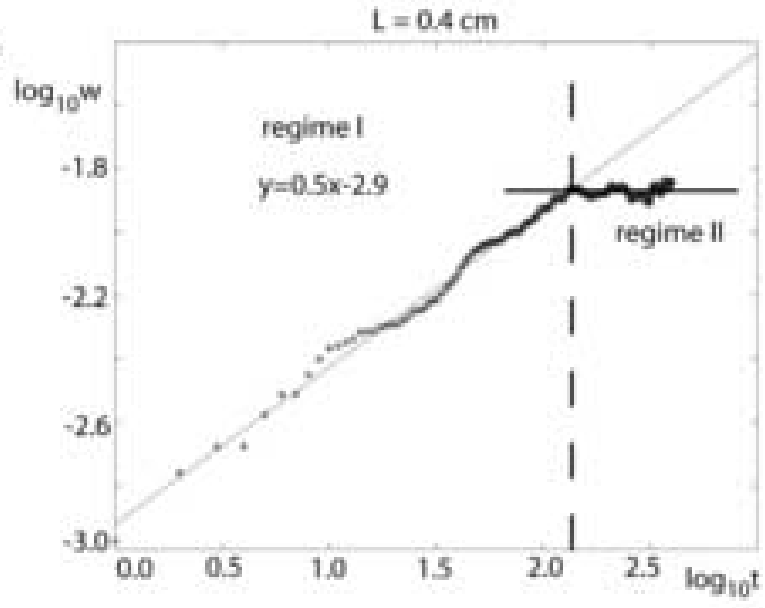

d)

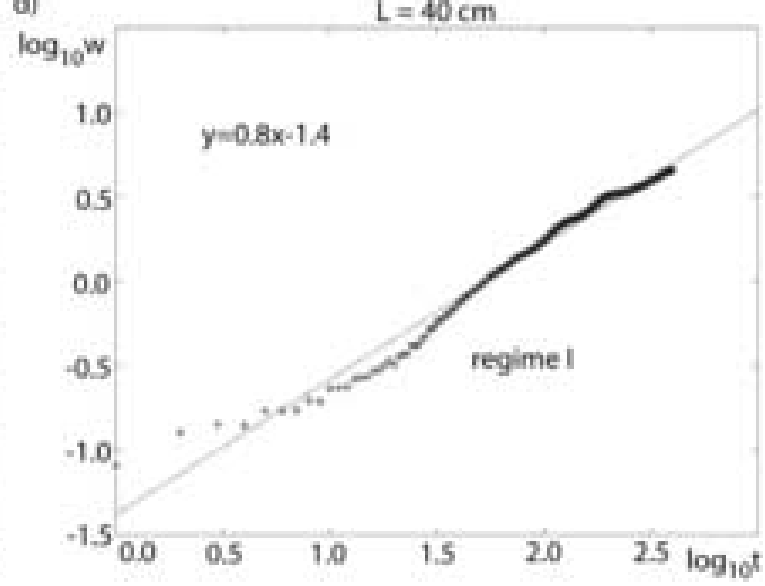

Fig. 6 
Click here to download high resolution image

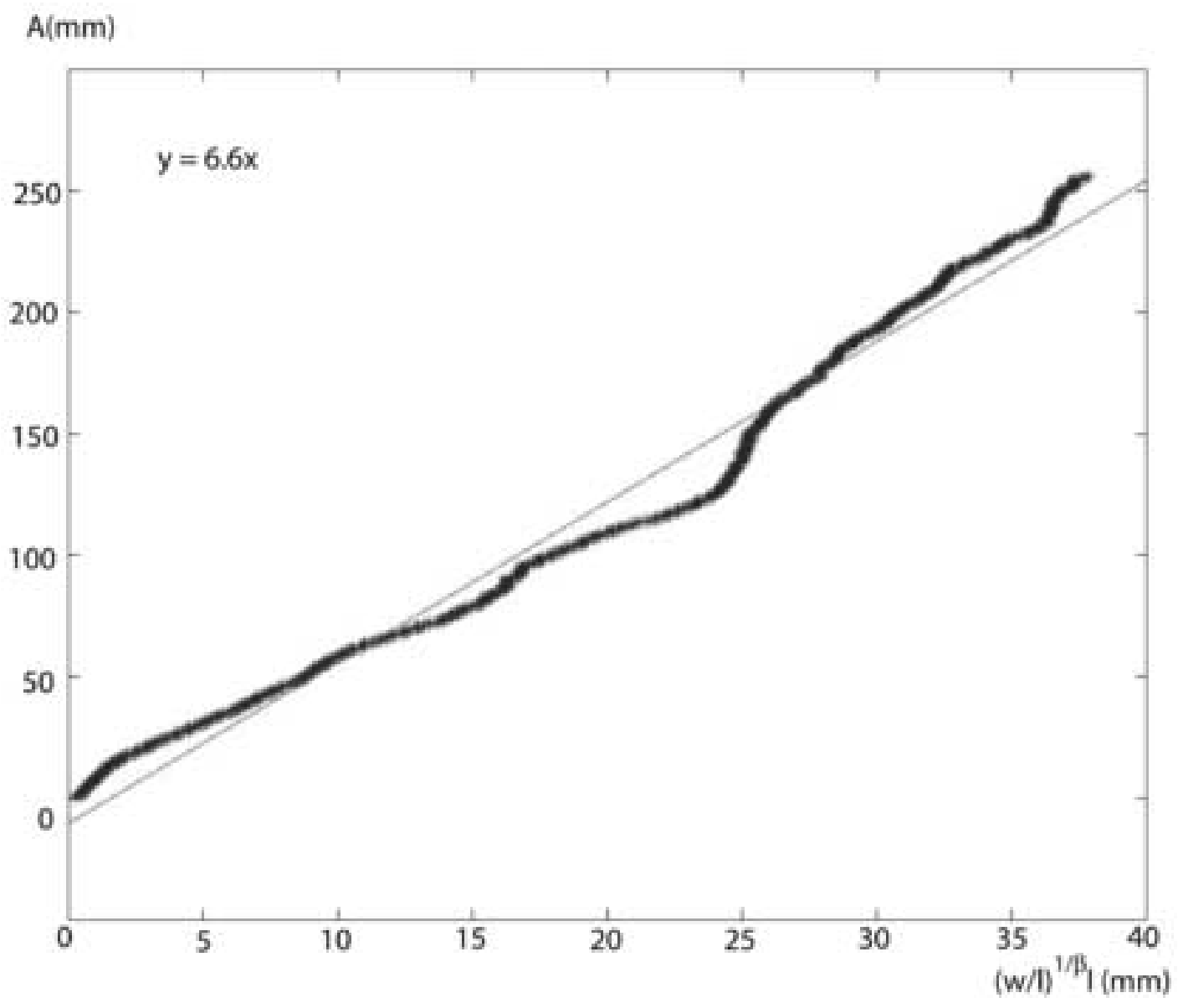

Fig. 7 


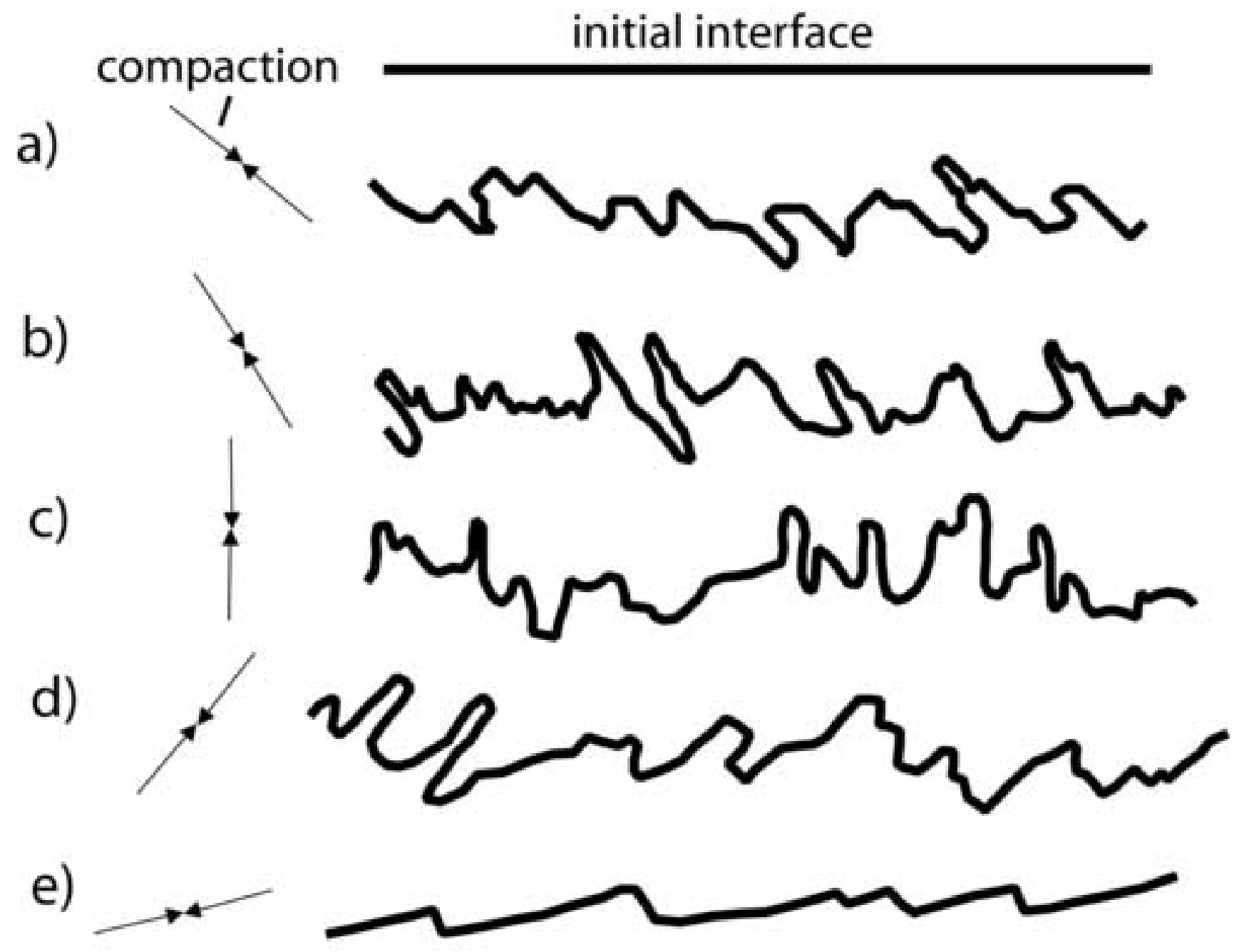

Fig. 8 
a)

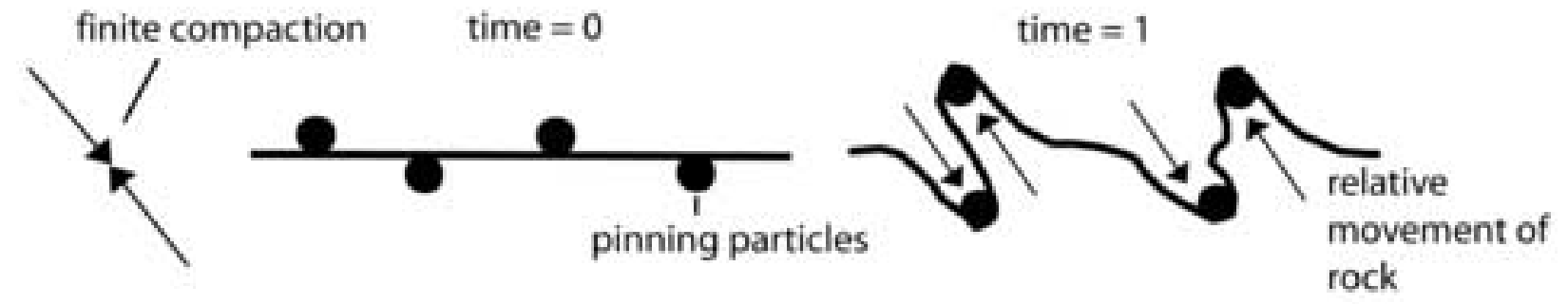

b) possible

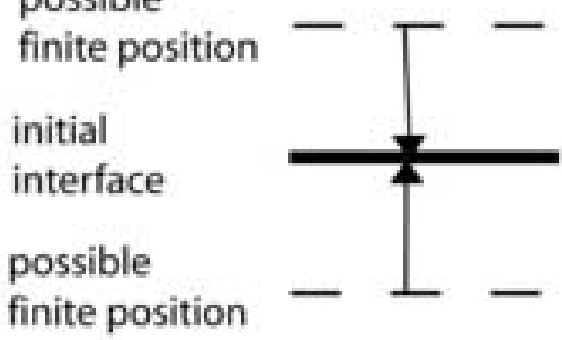

strong fluctuations

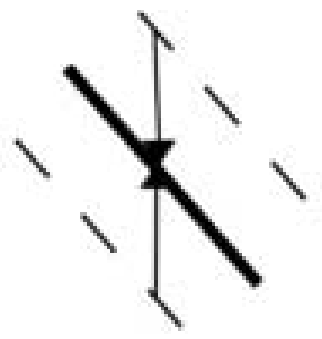

decreasing fluctuations

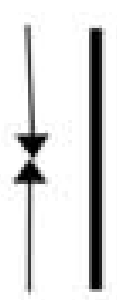

no fluctuations

c)

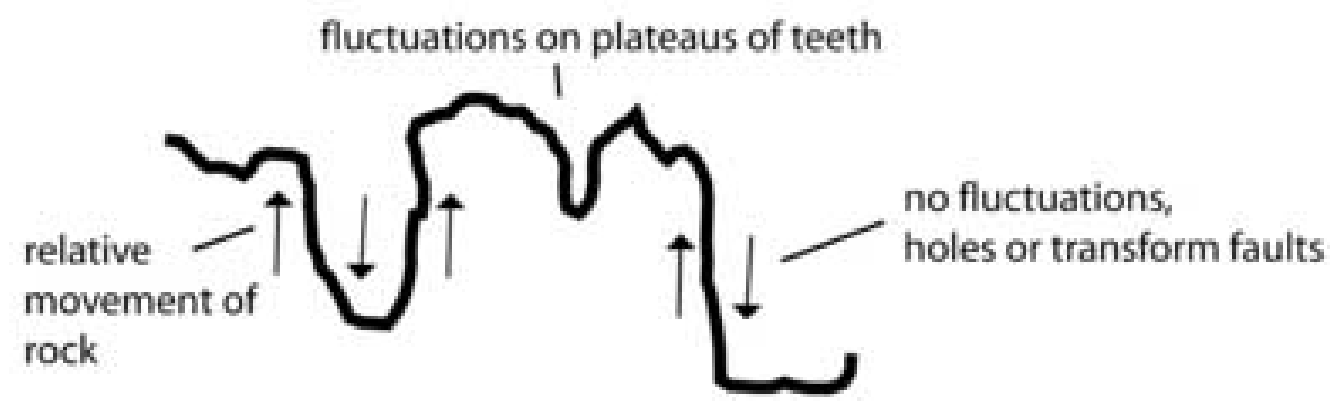

Fig. 9 